\title{
MANEUVERING AND CONTROL OF FLEXIBLE SPACE ROBOTS $\dagger$
}

\author{
Leonard Meirovitch* and Seungchul Lim** \\ Department of Engineering Science and Mechanics \\ Virginia Polytechnic Institute and State University \\ Blacksburg, VA 24061
}

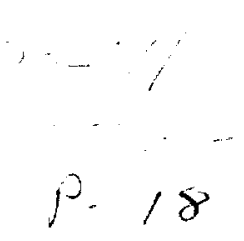

SUMMARY

This paper is concerned with a flexible space robot capable of maneuvering payloads. The robot is assumed to consist of two hinge-connected flexible arms and a rigid end-effector holding a payload; the robot is mounted on a rigid platform floating in space. The equations of motion are nonlinear and of high order. Based on the assumption that the maneuvering motions are one order of magnitude larger than the elastic vibrations, a perturbation approach permits design of controls for the two types of motion separately. The rigid-body maneuvering is carried out open loop, but the elastic motions are controlled closed loop, by means of discrete-time linear quadratic regulator theory with prescribed degree of stability. A numerical example demonstrates the approach. In the example, the controls derived by the perturbation approach are applied to the original nonlinear system and errors are found to be relatively small.

\section{INTRODUCTION}

A variety of space missions can be carried out effectively by space robots. These missions include the collection of space debris, recovery of spacecraft stranded in a useless orbit, repair of malfunctioning spacecraft, construction of a space station in orbit and servicing the space station while in operation. To maximize the usefulness of the space robot, the manipulator arms should be reasonably long. On the other hand, because of weight limitations, they must be relatively light. To satisfy both requirements, the manipulator arms must be highly flexible. Hence, space robots share some of the dynamics and control technology with articulated space structures.

Robotics has been an active research area for the past few decades, but applications have been concerned primarily with industrial robots, which are ground based and tend to be very stiff and bulky. In contrast, space robots are based on a floating platform and tend to be highly flexible. Hence, whereas industrial and space robots have a number of things in common, the differences are significant. More recent investigations have been concerned with flexible industrial robots (Refs. 1-4). On the other hand, some investigations are concerned with space robots consisting of rigid links (Refs. 5-7). Research on flexible space robots has come to light only recently (Refs. 8,9).

$\dagger$ Research supported by the NASA Research Grant NAG-1-225 monitored by Dr. R. C. Montgomery. The support is greatly appreciated.

* University Distinguished Professor

** Graduate Research Assistant 
This paper is concerned with a flexible space robot capable of maneuvering payloads. The robot is assumed to consist of two hinge-connected flexible arms and a rigid end-effector holding a payload; the robot is mounted on a rigid platform floating in space (Fig. 1). The platform is capable of translations and rotations, the flexible arms are capable of rotations and elastic deformations and the end-effector/payload can undergo rotations relative to the connecting flexible arm. Based on a consistent kinematical synthesis, the motions of one body in the chain take into consideration the motions of the preceding body in the chain. This permits the derivation of the equations of motion without the imposition of constraints. The equations of motion are derived by the Lagrangian approach. The equations are nonlinear and of relatively high order.

Ideally, the maneuvering of payloads should be carried without exciting elastic vibration, which is not possible in general. However, the elastic motions tend to be small compared to the rigid-body maneuvering motions. Under such circumstances, a perturbation approach permits separation of the problem into a zero-order problem (in a perturbation theory sense) for the rigid-body maneuvering of the space robot and a first-order problem for the control of the elastic motions and the perturbations from the rigid-body motions. The maneuvering can be carried out open loop, but the elastic and rigid-body perturbations are controlled closed loop.

The robot mission consists of carrying a payload over a prescribed trajectory and placing it in a certain orientation relative to the inertial space. For planar motion, the end-effector/payload configuration is defined by three variables, two translations and one rotation. At the end of the mission, the vibration should be damped out, so that the robot can be regarded as rigid at that time. Still, the rigid robot possesses six degrees of freedom, two translations of the platform and one rotation of each of the four bodies, including the platform. This implies that a kinematic redundancy exists. This redundancy is removed in the trajectory planning so as to conserve fuel. For a given end-effector/payload trajectory, the rigid-body maneuvering configuration of the robot can be obtained by means of inverse kinematics. Then, the forces and torques required for the robot trajectory realization are obtained from the zero-order equations by means of inverse dynamics.

The first-order equations for the elastic motions and the perturbations in the rigid-body maneuvering motions are linear, but of high order, time-varying and they are subjected to persistent disturbances. The persistent disturbances are treated by means of feedforward control. All other disturbances are controlled closed loop, with the feedback controls being designed by means of discrete-time linear quadratic regulator (LQR) theory with prescribed degree of stability. A numerical example demonstrates the approach. In the example, the controls derived by the perturbation approach are applied to the original nonlinear system and the errors in the endeffector/payload configuration were found to be relatively small during the maneuver and to vanish soon after the termination of the maneuver.

\section{A CONSISTENT KINEMATICAL SYNTHESIS}

To describe the motion of the space robot, it is convenient to adopt a consistent kinematical synthesis whereby the system is regarded as a chain of bodies and the motion of one body is 
defined with due consideration to the motion of the preceeding body in the chain. Figure 1 shows the mathematical model of the space robot, consisting of a rigid platform (Body 1), two hingeconnected flexible arms (Bodies 2 and 3) and a rigid end-effector holding the payload (Body 4). The various motions are referred to a set of inertial axes and sets of body axes to be defined shortly.

The object is to derive the system equations of motion, which can be done by means of Lagrange's equations in terms of quasi-coordinates (Ref. 10). Because in the case at hand the motion is planar, it is more expedient to use the standard Lagrange's equations. This requires the kinetic energy, potential energy and virtual work. The kinetic energy, in turn, requires the velocity of a typical point in each of the bodies.

The position of a nominal point on the platform is given by

$$
\mathbf{R}_{1}=\mathbf{R}_{0}+\mathbf{r}_{1}
$$

where $\mathbf{R}_{0}=\left[\begin{array}{ll}X Y & Y\end{array}\right]^{T}$ is the position vector of the origin $O_{1}$ of the body axes $x_{1}, y_{1}$ (Fig. 1) relative to the inertial axes $X, Y$ and in terms of $X, Y$ components and $r_{1}=\left[\begin{array}{ll}x_{1} & y_{1}\end{array}\right]^{T}$ is the position vector of the nominal point on the platform relative to the body axes $x_{1}, y_{1}$ and in terms of $x_{1}, y_{1}$ components. The velocity vector of a point on the platform can be expressed in terms of $x_{1}, y_{1}$ components as follows:

$$
V_{1}=C_{1} \dot{\mathbf{R}}_{0}+\tilde{\omega}_{1} \mathbf{r}_{1}
$$

where

$$
C_{1}=\left[\begin{array}{cc}
c \theta_{1} & s \theta_{1} \\
-s \theta_{1} & c \theta_{1}
\end{array}\right]
$$

is a matrix of direction cosines between axes $x_{1}, y_{1}$, and $X, Y$, in which $s \theta_{1}=\sin \theta_{1}, c \theta_{1}=\cos \theta_{1}$,

$$
\dot{\mathbf{R}}_{0}=\left[\begin{array}{ll}
\dot{X} & \dot{Y}
\end{array}\right]^{T}
$$

is the velocity vector of $O_{1}$ in terms of $X, Y$ components and

$$
\tilde{\omega}_{1}=\left[\begin{array}{cc}
0 & -\dot{\theta}_{1} \\
\dot{\theta}_{1} & 0
\end{array}\right]
$$

The second body is flexible, so that we must resolve the question of body axes. We define the body axes $x_{2}, y_{2}$ as a set of axes with the origin at the hinge $\mathrm{O}_{2}$ and embedded in the undeformed body such that $x_{2}$ is tangent to the body at $\mathrm{O}_{2}$ (Fig. 2). Then, we define the motion of axes $x_{2}, y_{2}$ as rigid-body motion and measure the elastic motion relative to $x_{2}, y_{2}$. Hence, the velocity of a point in the second body (first flexible arm) in terms of $x_{2}, y_{2}$ components is

$$
\begin{aligned}
\mathbf{V}_{2} & =C_{2-1} \mathbf{V}_{1}\left(O_{2}\right)+\tilde{\omega}_{2}\left(\mathbf{r}_{2}+\mathbf{u}_{2}\right)+\dot{\mathbf{u}}_{2 \mathrm{rel}} \\
& =C_{2} \dot{\mathbf{R}}_{0}+C_{2-1} \tilde{\omega}_{1} \mathbf{r}_{1}\left(O_{2}\right)+\tilde{\omega}_{2}\left(\mathbf{r}_{2}+\mathbf{u}_{2}\right)+\dot{\mathbf{u}}_{2 \mathrm{rel}}
\end{aligned}
$$

where $C_{2-1}$ and $C_{2}$ are matrices similar to $C_{1}$, Eq. (3), except that $\theta_{1}$ is replaced by $\theta_{2}-\theta_{1}$ and $\theta_{2}$, respectively, $\tilde{\omega}_{2}$ has the same structure as $\tilde{\omega}_{1}$ but with $\dot{\theta}_{2}$ replacing $\dot{\theta}_{1}, \mathbf{r}_{1}\left(O_{2}\right)=\left[d_{1} h_{1}\right]^{T}, \mathbf{r}_{2}=$ 
$\left[\begin{array}{ll}x_{2} & 0\end{array}\right]^{T}, \mathrm{u}_{2}=\left[\begin{array}{ll}0 & u_{2}\end{array}\right]^{T}$ and $\dot{\mathrm{u}}_{2 \mathrm{rel}}=\left[\begin{array}{ll}0 & \dot{u}_{2}\end{array}\right]$, in which $u_{2}=u_{2}\left(x_{2}, t\right)$ and $\dot{u}_{2}=\dot{u}_{2}\left(x_{2}, t\right)$ are the elastic displacement and velocity, respectively.

Using the analogy with the second body, the velocity of a point in the third body (second flexible arm) in terms of $x_{3}, y_{3}$ components can be shown to be

$$
\begin{aligned}
\mathbf{V}_{3}= & C_{3-2} \mathbf{V}_{2}\left(L_{2}\right)+\tilde{\omega}_{3}\left(\mathbf{r}_{3}+\mathbf{u}_{3}\right)+\dot{\mathbf{u}}_{3 \mathrm{rel}} \\
= & C_{3} \dot{\mathbf{R}}_{0}+C_{3-1} \tilde{\omega}_{1} \mathbf{r}_{1}\left(O_{2}\right)+C_{3-2}\left\{\tilde{\omega}_{2}\left[\mathbf{r}_{2}\left(L_{2}\right)+\mathbf{u}_{2}\left(L_{2}, t\right)\right]+\dot{\mathbf{u}}_{2 \mathrm{rel}}\left(L_{2}, t\right)\right\} \\
& +\tilde{\omega}_{3}\left(\mathbf{r}_{3}+\mathbf{u}_{3}\right)+\dot{\mathbf{u}}_{3 \mathrm{rel}}
\end{aligned}
$$

The fourth body consists of the end-effector and payload combined, and is treated as rigid. Following the established pattern, the velocity of a point in the fourth body in terms of $x_{4}, y_{4}$ components is

$$
\begin{aligned}
\mathbf{V}_{4}= & C_{4-3} \mathbf{V}_{3}\left(L_{3}\right)+\tilde{\omega}_{4} \mathbf{r}_{4} \\
= & C_{4} \dot{\mathbf{R}}_{0}+C_{4-1} \tilde{\omega}_{1} \mathbf{r}_{1}\left(O_{2}\right)+C_{1-2}\left\{\tilde{\omega}_{2}\left[\mathbf{r}_{2}\left(L_{2}\right)+\mathbf{u}_{2}\left(L_{2}, t\right)\right]+\dot{\mathbf{u}}_{2 \mathrm{rel}}\left(L_{2}, t\right)\right\} \\
& +C_{4-3}\left\{\tilde{\omega}_{3}\left[\mathbf{r}_{3}\left(L_{3}\right)+\mathbf{u}_{3}\left(L_{3}, t\right)\right]+\dot{\mathbf{u}}_{3 \mathrm{rel}}\left(L_{3}, t\right)\right\}+\tilde{\omega}_{4} \mathbf{r}_{4}
\end{aligned}
$$

The consistent kinematical synthesis just described permits the formulation of the equations of motion for the whole system without invoking constraint equations. Such constraint equations must be used to eliminate redundant coordinates in a formulation in which equations of motion are derived separately for each body.

\section{SPATIAL DISCRETIZATION OF THE FLEXIBLE ARMS}

The velocity expressions derived in Sec. 2 involve rigid-body motions depending on time alone and elastic motions depending on the spatial position and time. Equations of motion based on such formulations are hybrid, in the sense that the equations for the rigid-body motions are ordinary differential equations and the ones for the elastic motions are partial differential equations. Designing maneuvers and controls on the basis of hybrid differential equations is likely to cause serious difficulties, so that the only viable alternative is to transform the hybrid system into one consisting of ordinary differential equations alone. This amounts to discretization in space of the elastic displacements, which can be done by means of series expansions. Assuming that the flexible arms act as beams in bending, the elastic displacements can be expanded in the series

$$
u_{i}\left(x_{i}, t\right)=\sum_{j=1}^{n_{i}} \phi_{i j}\left(x_{i}\right) \eta_{i j}(t)=\phi_{i}^{T}\left(x_{i}\right) \eta_{i}(t), i=2,3
$$

where $\phi_{i j}\left(x_{i}\right)$ are admissible functions, often referred to as shape functions, and $\eta_{i j}(t)$ are generalized coordinates; $\phi_{j}$ and $\eta_{i}$ are corresponding $n_{i}$-dimensional vectors $(i=2,3 ; j=$ $\left.1,2, \ldots, n_{i}\right)$

The question arises as to the nature of the admissible functions. Clearly, the object is to approximate the displacements with as few terms in the series as possible. This is not a new 
problem in structural dynamics, and the very same subject has been investigated recently in Ref. 11 , in which a new class of functions, referred to as quasi-comparison functions, has been introduced. Quasi-comparison functions are defined as linear combinations of admissible functions capable of satisfying the boundary conditions of the elastic member. As shown in Fig. 2, the beam is tangent to axis $x_{i}$ at $O_{i}(i=2,3)$. Hence, the admissible functions must be zero and their slope must be zero at $x_{i}=0$. At $x_{i}=L_{i}$, the displacement, slope, bending moment and shearing force are generally nonzero. Quasi-comparison functions are linear combinations of functions possessing these characteristics. Admissible functions from a single family of functions do not possess the characteristics, but admissible functions from several suitable families can be combined to obtain them. In the case at hand, quasi-comparison functions can be obtained in the form of suitable linear combinations of clamped-free and clamped-clamped shape functions.

\section{LAGRANGE'S EQUATIONS}

Before we can derive Lagrange's equations, we must produce expressions for the kinetic energy, potential energy and virtual work. To this end, and following the spatial discretization indicated by Eqs. (9), we introduce the configuration vector

$$
\mathrm{q}(t)=\left[X(t) Y(t) \theta_{1}(t) \theta_{2}(t) \theta_{3}(t) \theta_{4}(t) \eta_{2}^{T}(t) \eta_{3}^{T}(t)\right]^{T}
$$

so that the velocity vectors, Eqs. (2), (6)-(8), can be written in the compact form

$$
\mathrm{V}_{\mathrm{i}}=D_{\mathrm{i}} \dot{\mathrm{q}}, i=1,2,3,4
$$

where

$$
\begin{aligned}
D_{1} & =\left[\begin{array}{cccccc}
c \theta_{1} & s \theta_{1} & -y_{1} & 0 & \ldots & 0^{T} \\
-s \theta_{1} & c \theta_{1} & x_{1} & 0 & \ldots & 0^{T}
\end{array}\right] \\
D_{2} & =\left[\begin{array}{cccccccc}
c \theta_{2} & s \theta_{2} & d_{1} s\left(\theta_{2}-\theta_{1}\right)-h_{1} c\left(\theta_{2}-\theta_{1}\right) & -\phi_{2}^{T} \eta_{2} & 0 & 0 & 0^{T} & 0^{T} \\
-s \theta_{2} & c \theta_{2} & d_{1} c\left(\theta_{2}-\theta_{1}\right)+h_{1} s\left(\theta_{2}-\theta_{1}\right) & x_{2} & 0 & 0 & \phi_{2}^{T} & 0^{T}
\end{array}\right]
\end{aligned}
$$

Then, the kinetic energy is simply

$$
T=\frac{1}{2} \sum_{i=1}^{4} \int_{m_{i}} \mathbf{V}_{i}^{T} \mathbf{V}_{i} d m_{i}=\frac{1}{2} \dot{\mathbf{q}}^{T} M \dot{\mathbf{q}}
$$

where

$$
M=\sum_{i=1}^{4} \int_{m_{i}} D_{i}^{T} D_{i} d m_{i}
$$

is the mass matrix. Typical entries in the mass matrix are

$$
\begin{aligned}
& m_{11}=m, m_{12}=0, m_{13}=-\left(m_{2}+m_{3}+m_{4}\right)\left(h_{1} c \theta_{1}+d_{1} s \theta_{1}\right) \\
& m_{14}=-\left[\bar{\phi}_{2}^{T}+\left(m_{3}+m_{4}\right) \phi_{2}^{T}\left(L_{2}\right)\right] \eta_{2} c \theta_{2}-\left[S_{2}+\left(m_{3}+m_{4}\right) L_{2}\right] s \theta_{2}
\end{aligned}
$$




$$
\begin{aligned}
& m_{18}=-\left[\bar{\phi}_{3}^{T}+m_{4} \phi_{3}^{T}\left(L_{3}\right)\right] s \theta_{3} \\
& m_{22}=m, m_{23}=-\left(m_{2}+m_{3}+m_{4}\right)\left(h_{1} s \theta_{1}-d_{1} c \theta_{1}\right) \\
& m_{28}=\left[\bar{\phi}_{3}^{T}+m_{4} \phi_{3}^{T}\left(L_{3}\right)\right] c \theta_{3} \\
& m_{88}=\int_{\text {Body } 3} \phi_{3} \phi_{3}^{T} d m_{3}+m_{4} \phi_{3}\left(L_{3}\right) \phi_{3}^{T}\left(L_{3}\right)
\end{aligned}
$$

in which

$$
m=\sum_{i=1}^{4} m_{i}, \bar{\phi}_{i}=\int_{m_{i}} \phi_{i} d m_{i}, i=2,3, S_{i}=\int_{m_{i}} x_{i} d m_{i}, i=1,2,3,4
$$

The potential energy, assumed to be entirely due to bending, has the form

$$
V=\frac{1}{2} \int_{0}^{L_{2}} E I_{2}\left[u_{2}^{\prime \prime}\left(x_{2}, t\right)\right]^{2} d x_{2}+\frac{1}{2} \int_{0}^{L_{3}} E I_{3}\left[u_{3}^{\prime \prime}\left(x_{3}, t\right)\right]^{2} d x_{3}=\frac{1}{2} \mathbf{q}^{T} K \mathbf{q}
$$

in which $E I_{i}(i=2,3)$ are bending stiffnesses and primes denote spatial derivatives. Moreover,

$$
K=\text { block }-\operatorname{diag}\left[\begin{array}{lll}
0 & K_{2} & K_{3}
\end{array}\right]
$$

is the stiffness matrix, where

$$
K_{i}=\int_{0}^{L_{i}} E I_{i} \phi_{i}^{\prime \prime}\left(\phi_{i}^{\prime \prime}\right)^{T} d x_{i}, i=2,3
$$

are stiffness matrices for the flexible arms.

Next, we propose to derive the virtual work expression. To this end, we must specify first the actuators to be used. There are three actuators acting on the platform, two thrusters $F_{x 1}$ and $F_{y 1}$ acting in directions aligned with the body axes and a torquer $M_{1}$ acting at $O_{1}$. Three other torquers $M_{2}, M_{3}$ and $M_{4}$ are located at the hinges $O_{2}, O_{3}$ and $O_{4}$, respectively, the first acting on the platform and first arm, the second acting on the first and second arm and the third acting on the second arm and end-effector. In view of this, the virtual work can be written as follows:

$$
\begin{aligned}
\delta W= & F_{x 1}\left(\cos \theta_{1} \delta X+\sin \theta_{1} \delta Y\right)+F_{y 1}\left(-\sin \theta_{1} \delta X+\cos \theta_{1} \delta Y\right)+M_{1} \delta \theta_{1} \\
& +M_{2} \delta\left(\theta_{2}-\theta_{1}\right)+M_{3} \delta \psi_{3}+M_{4} \delta \psi_{4}+M_{5} \delta\left[\theta_{2}+\phi_{2}^{\prime T}\left(L_{2} / 3\right) \eta_{2}\right] \\
& +M_{6} \delta\left[\theta_{2}+\phi_{2}^{\prime T}\left(2 L_{2} / 3\right) \eta_{2}\right]+M_{7} \delta\left[\theta_{3}+\phi_{3}^{\prime T}\left(L_{3} / 3\right) \eta_{3}\right]+M_{8}\left[\theta_{3}+\phi_{3}^{\prime T}\left(2 L_{3} / 3\right) \eta_{3}\right]
\end{aligned}
$$

where $\delta X, \delta Y, \ldots$ are virtual displacements. Moreover, denoting the angles between the two arms and between the second arm and the end-effector by

$$
\begin{aligned}
& \psi_{3}=\theta_{3}-\theta_{2}-\left.\frac{\partial u_{2}}{\partial x_{2}}\right|_{x_{2}=L_{2}}=\theta_{3}-\theta_{2}-\phi_{2}^{\prime T}\left(L_{2}\right) \eta_{2} \\
& \psi_{4}=\theta_{4}-\theta_{3}-\left.\frac{\partial u_{3}}{\partial x_{3}}\right|_{x_{3}=L_{3}}=\theta_{4}-\theta_{3}-\phi_{3}^{\prime T}\left(L_{3}\right) \eta_{3}
\end{aligned}
$$


we can write

$$
\delta \psi_{3}=\delta \theta_{3}-\delta \theta_{2}-\phi_{2}^{T}\left(L_{2}\right) \delta \eta_{2}, \delta \psi_{4}=\delta \theta_{4}-\delta \theta_{3}-\phi_{3}^{r T}\left(L_{3}\right) \delta \eta_{3}
$$

Inserting Eqs. (22) into Eq. (20), we can express the virtual work in terms of generalized forces and generalized virtual displacements in the form

$$
\delta W=\mathbf{Q}^{T} \delta \mathbf{q}
$$

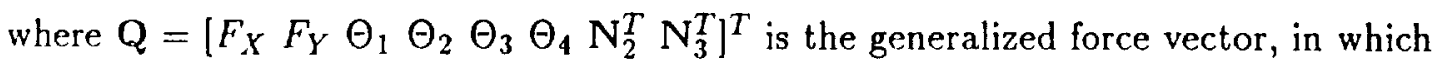

$$
\begin{aligned}
F_{X} & =F_{x 1} \cos \theta_{1}-F_{y 1} \sin \theta_{1}, F_{Y}=F_{x 1} \sin \theta_{1}+F_{y 1} \cos \theta_{1} \\
\Theta_{1} & =M_{1}-M_{2}, \Theta_{2}=M_{2}-M_{3}+M_{5}+M_{6} \\
\Theta_{3} & =M_{3}-M_{4}+M_{7}+M_{8}, \Theta_{4}=M_{4} \\
N_{2} & =M_{5} \phi_{2}^{\prime}\left(L_{2} / 3\right)+M_{6} \phi_{2}^{\prime}\left(2 L_{2} / 3\right)-M_{3} \phi_{2}^{\prime}\left(L_{2}\right) \\
\mathrm{N}_{3} & =M_{7} \phi_{3}^{\prime}\left(L_{3} / 3\right)+M_{8} \phi_{3}^{\prime}\left(2 L_{3} / 3\right)-M_{4} \phi_{3}^{\prime}\left(L_{3}\right)
\end{aligned}
$$

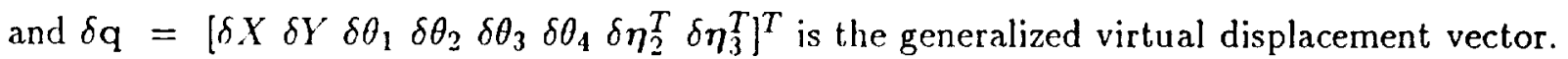
Equations (24) express the generalized forces and torques in terms of the actual actuator forces and torques and can be expressed in the compact form

$$
\mathrm{Q}=E \mathrm{~F}
$$

where $\mathbf{F}=\left[\begin{array}{llllll}F_{x 1} & F_{y 1} & M_{1} & M_{2} & \ldots & M_{8}\end{array}\right]^{T}$ is the actual control vector and

$E=E\left(\theta_{1}\right)=$
$\left[\begin{array}{cccccccccc}\cos \theta_{1} & -\sin \theta_{1} & 0 & 0 & 0 & 0 & 0 & 0 & 0 & 0 \\ \sin \theta_{1} & \cos \theta_{1} & 0 & 0 & 0 & 0 & 0 & 0 & 0 & 0 \\ 0 & 0 & 1 & -1 & 0 & 0 & 0 & 0 & 0 & 0 \\ 0 & 0 & 0 & 1 & -1 & 0 & 1 & 1 & 0 & 0 \\ 0 & 0 & 0 & 0 & 1 & -1 & 0 & 0 & 1 & 1 \\ 0 & 0 & 0 & 0 & 0 & 1 & 0 & 0 & 0 & 0 \\ \mathbf{0} & \mathbf{0} & \mathbf{0} & \mathbf{0} & -\phi_{2}^{\prime}\left(L_{2}\right) & \mathbf{0} & \phi_{2}^{\prime}\left(\frac{L_{2}}{3}\right) & \phi_{2}^{\prime}\left(\frac{2 L_{2}}{3}\right) & 0 & 0 \\ \mathbf{0} & \mathbf{0} & \mathbf{0} & \mathbf{0} & \mathbf{0} & -\phi_{3}^{\prime}\left(L_{3}\right) & \mathbf{0} & \mathbf{0} & \phi_{3}^{\prime}\left(\frac{L_{3}}{3}\right) & \phi_{3}^{\prime}\left(\frac{2 L_{3}}{3}\right)\end{array}\right]$

is a time-varying coefficient matrix, because $\theta_{1}$ varies with time.

Lagrange's equation can be expressed in the general symbolic vector form

$$
\frac{d}{d t}\left(\frac{\partial T}{\partial \dot{q}}\right)-\frac{\partial T}{\partial \mathbf{q}}+\frac{\partial V}{\partial \mathbf{q}}=\mathbf{Q}
$$


Observing that $M=M(\mathrm{q})$, we can write

$$
\begin{aligned}
& \frac{\partial T}{\partial \dot{\mathrm{q}}}=M \dot{\mathrm{q}}, \frac{d}{d t}\left(\frac{\partial T}{\partial \dot{\mathrm{q}}}\right)=M \ddot{\mathrm{q}}+\dot{M} \dot{\mathrm{q}} \\
& \frac{\partial T}{\partial \mathrm{q}}=\frac{1}{2} \dot{\mathrm{q}}^{T} \frac{\partial M}{\partial \mathrm{q}} \dot{\mathrm{q}}, \frac{\partial V}{\partial \mathrm{q}}=K \mathrm{q}
\end{aligned}
$$

Inscrting Eqs. (28) into Eq. (27), we obtain Lagrange's equations in the more explicit form

$$
M \ddot{\mathrm{q}}+\left(\dot{M}-\frac{1}{2} \dot{\mathrm{q}}^{T} \frac{\partial M}{\partial \mathrm{q}}\right) \dot{\mathrm{q}}+K \mathbf{q}=\mathbf{Q}
$$

in which

$$
\dot{M}=\sum_{j=1}^{6+2 n} \frac{\partial M}{\partial q_{j}} \dot{q}_{j}, \dot{\mathrm{q}}^{T} \frac{\partial M}{\partial \mathrm{q}}=\left[\begin{array}{c}
\dot{\mathrm{q}}^{T} \partial M / \partial q_{1} \\
\dot{\mathrm{q}}^{T} \partial M / \partial q_{2} \\
\vdots \\
\dot{\mathrm{q}}^{T} \partial M / \partial q_{6+2 n}
\end{array}\right]
$$

\section{A PERTURBATION APPROACH TO THE CONTROL DESIGN}

Equation (29) represents a high-order system of nonlinear differential equations, and is not very suitable for control design. Hence, an approach capable of coping with the problems of highdimensionality and nonlinearity is highly desirable. Such an approach must be based on the physics of the problem. The ideal maneuver is that in which the robot acts as if its arms were rigid. In reality, the arms are flexible, so that some elastic vibrations are likely to take place. It is reasonable to assume, however, that the elastic motions are one order of magnitude smaller than the maneuvering motions. This permits treatment of the elastic motions as perturbations on the maneuvering motions. In turn, the elastic perturbations give rise to perturbations in the "rigid-body" maneuvering motions. This suggests a perturbation approach, whereby the problem is separated into a zero-order problem for the "rigid-body" maneuvering of the payload and a first-order problem for the control of the elastic motions and the perturbations in the rigid-body maneuvering motions. The zero-order problem is nonlinear, albeit of relatively low dimension. It can be solved independently and the control can be open loop. On the other hand, the first-order problem is linear, but of relatively high dimension. It is affected by the solution to the zero-order problem, where the effect is in the form of time-varying coefficients and persistent disturbances. The control for the first-order problem is to be closed loop.

We consider a first-order perturbation solution characterized by

$$
\mathbf{q}=\mathrm{q}_{0}+\mathrm{q}_{1}, \mathbf{Q}=\mathbf{Q}_{0}+\mathbf{Q}_{1}
$$

where the subscripts 0 and 1 denote zero-order and first-order quantities, with the zero-order quantities being one order of magnitude larger than the first-order ones. Inserting Eqs. (31) into Eq. (29) and separating quantities of different orders of magnitude, we obtain the equation for the zero-order problem

$$
M_{0} \ddot{\mathrm{q}}_{0}+\left(M_{v}-\frac{1}{2} M_{v}^{T}\right) \dot{\mathrm{q}}_{0}=\mathbf{Q}_{0}=E_{0} \mathbf{F}_{0}
$$




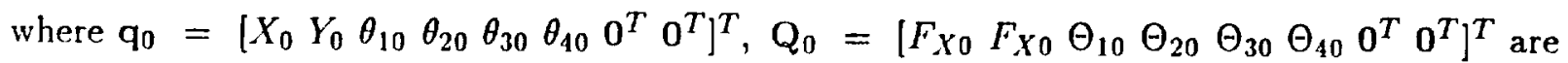
zero-order displacement and generalized control vectors, $E_{0}=E\left(\theta_{10}\right)$ is the matrix $E$, Eq. (26),

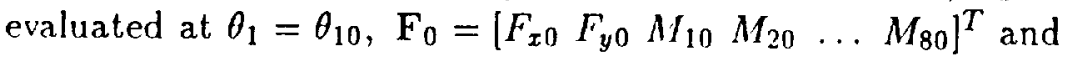

$$
M_{0}=M\left(\mathbf{q}_{0}\right), M_{v}=\left.\left[\begin{array}{llll}
\frac{\partial M}{\partial q_{1}} \dot{\mathrm{q}}_{0} & \frac{\partial M}{\partial q_{2}} \dot{\mathrm{q}}_{0} & \ldots & \frac{\partial M}{\partial q_{6+2 n}} \dot{\mathrm{q}}_{0}
\end{array}\right]\right|_{\mathrm{q}=\mathrm{q}_{0}}
$$

Moreover, we obtain the equation for the first-order problem

$$
M_{0} \ddot{\mathrm{q}}_{1}+\left(M_{v}+M^{\prime}-M_{v}^{T}\right) \dot{\mathrm{q}}_{1}+\left(M_{a}+M_{v v}-\frac{1}{2} M_{v v}^{\prime}+K\right) \mathrm{q}_{1}=\mathrm{Q}_{1}+\mathrm{Q}_{d}
$$

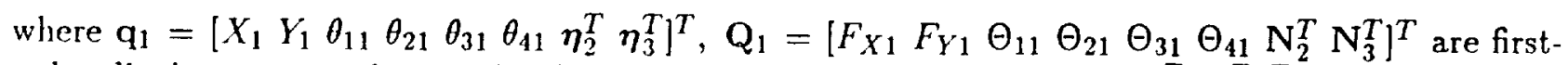
order displacement and generalized control vectors, $Q_{d}=\left[\begin{array}{llllllllll}0 & 0 & 0 & 0 & 0 & 0 & F_{d 2}^{T} & F_{d 3}^{T}\end{array}\right]^{T}$ is a persistent disturbance vector and

$$
\begin{aligned}
& M_{a}=\left.\left[\begin{array}{llll}
\frac{\partial M}{\partial q_{1}} \ddot{\mathbf{q}}_{0} & \frac{\partial M}{\partial q_{2}} \ddot{\mathrm{q}}_{0} & \ldots & \frac{\partial M}{\partial q_{6+2 n}} \ddot{\mathbf{q}}_{0}
\end{array}\right]\right|_{\mathbf{q}=\mathbf{q}_{0}} \\
& M^{\prime}=\left.\sum_{j=1}^{6+2 n} \frac{\partial M}{\partial q_{j}}\right|_{\mathbf{q}=q_{0}} \dot{\mathrm{q}}_{0 j} \\
& M_{v v} \mathrm{q}_{1}=\left.\sum_{j=1}^{6+2 n} \sum_{k=1}^{6+2 n} \frac{\partial^{2} M}{\partial q_{j} \partial q_{k}}\right|_{\mathbf{q}=q_{0}} q_{1 k} \dot{q}_{0 j} \dot{\mathrm{q}}_{0} \\
& M_{v v}^{\prime} \mathbf{q}_{1}=\left.\dot{\mathbf{q}}_{0}^{T} \sum_{k=1}^{6+2 n} \frac{\partial^{2} M}{\partial \mathrm{q} \partial q_{k}}\right|_{\mathbf{q}=\mathbf{q}_{0}} q_{1 k} \dot{\mathrm{q}}_{0}
\end{aligned}
$$

From Eqs. (25) and (26), however, we can write

$$
\mathrm{Q}_{1}=E_{0} \mathbf{F}_{1}+E_{1} \mathbf{F}_{0}=E_{0} \mathbf{F}_{1}+\mathbf{F}_{0}^{*} \mathrm{q}_{1}
$$

where $E_{1}=\left[\partial E / \partial \theta_{1} \mid \theta_{1}=\theta_{10}\right] \theta_{11}$. Moreover, the matrix $F_{0}^{*}$ has the entries

$$
\begin{aligned}
& F_{011}^{*}=-\left(F_{x 10} \sin \theta_{10}+F_{y 10} \cos \theta_{10}\right) \\
& F_{021}^{*}=F_{x 10} \cos \theta_{10}-F_{y 10} \sin \theta_{10} \\
& F_{0 i j}^{*}=0, i=3,4, \ldots, 6+n_{2}+n_{3} ; j=2,3, \ldots, 6+n_{2}+n_{3}
\end{aligned}
$$

In view of this, the equation for the first-order problem can be rewritten as

$$
M_{0} \ddot{\mathrm{q}}_{1}+\left(M_{v}+M^{\prime}-M_{v}^{T}\right) \dot{\mathrm{q}}_{1}+\left(M_{a}+M_{v v}-\frac{1}{2} M_{v v}^{\prime}+K-F_{0}^{*}\right) \mathrm{q}_{1}=E_{0} \mathrm{~F}_{1}+\mathrm{Q}_{d}
$$

\section{TRAJECTORY PLANNING}

The mission consists of delivering the payload to a certain point in space and placing it in a certain orientation. For planar motion, the final payload configuration is defined by three variables, two translations and one rotation. The trajectory planning, designed to realize this 
final configuration, will be carried out as if the robot system were rigid, with the expectation that all elastic motions and perturbations in the rigid-body maneuvering motions will be annihilated by the end of the maneuver. The rigid-body motion of the robot is described by the zero-order problem and it consists of six components, two translations of the platform and one rotation of each of the four bodies. This implies that a kinematical redundancy exists, as there is an infinity of ways a six-dimensional configuration can generate a three-dimensional trajectory. This redundancy can be removed by controlling three of the configuration variables, such as the translations and rotation of the platform, so as to conserve fuel. Under these circumstances, the rigid space robot can be treated as a nonredundant manipulator.

Next, we denote the end-effector configuration by $\mathbf{X}_{E}$, so that from kinematics we can write

$$
\mathbf{X}_{E}=\mathbf{f}\left(\mathbf{q}_{0}\right)
$$

where $\mathbf{f}$ is a three-dimensional vector function. From differential kinematics, we have

$$
\dot{\mathrm{X}}_{E}=J\left(\mathrm{q}_{0}\right) \dot{\mathrm{q}}_{0}
$$

where

$$
J\left(\mathbf{q}_{0}\right)=\left[\partial \mathbf{f} / \partial \mathbf{q}_{0}\right]
$$

is the $3 \times 6 \mathrm{~J}$ acobian matrix. Introducing the notation

$$
\mathrm{q}_{0}=\left[\begin{array}{l:l}
\mathrm{q}_{S}^{T} & \mathbf{q}_{M}^{T}
\end{array}\right]^{T}
$$

where

$$
\mathrm{q}_{S}=\left[\begin{array}{lll}
X_{0} & Y_{0} & \theta_{10}
\end{array}\right]^{T}, \mathrm{q}_{M}=\left[\begin{array}{lll}
\theta_{20} & \theta_{30} & \theta_{\mathbf{4 0}}
\end{array}\right]^{T}
$$

are the controlled platform configuration vector and the open-loop controlled manipulator configuration vector, and partitioning the Jacobian matrix accordingly, or

$$
J=\left[\begin{array}{l|l}
J_{S} & J_{M}
\end{array}\right]
$$

Eq. (40) can be rewritten as

$$
\dot{\mathrm{X}}_{E}=J_{S} \dot{\mathrm{q}}_{S}+J_{M} \dot{\mathrm{q}}_{M}
$$

Then, on the assumption that $\dot{\mathrm{q}} S$ is determined so as to minimize the fuel consumption, and for a given end-effector trajectory $\mathrm{X}_{E}$, we can determine the manipulator velocity vector from

$$
\dot{\mathrm{q}}_{M}=J_{M}^{-1}\left(\dot{\mathrm{X}}_{E}-J_{S} \dot{\mathrm{q}}_{S}\right)
$$

The end-effector trajectory was taken in the form of a sinusoidal function so as to prevent excessive vibration. Finally, with $\mathbf{q}_{0}$ given, we can obtain the required open-loop control $F_{0}$ by inverse dynamics, which amounts to using Eq. (32). 


\section{FEEDBACK CONTROL OF THE ELASTIC MOTIONS AND RIGID-BODY PERTURBATIONS}

The elastic motions and the perturbations in the rigid-body maneuvering motions are governed by the equation defining the first-order problem, Eq. (38). The persistent disturbances are controlled open loop and all other disturbances are controlled closed loop. To this end, we express the control vector in the form

$$
\mathrm{F}_{1}=\mathrm{F}_{10}+\mathrm{F}_{1 \mathrm{c}}
$$

where the subscripts $o$ and $c$ indicate open loop and closed loop, respectively. Recognizing that $E_{0}$ is a rectangular matrix, the open-loop control can be written as

$$
\mathrm{F}_{1 o}=-E_{0}^{\dagger} \mathrm{Q}_{d}
$$

in which

$$
E_{0}^{\dagger}=\left(E_{0}^{T} E_{0}\right)^{-1} E_{0}^{T}
$$

is the psuedo-inverse of $E_{0}$.

For the closed-loop control, we consider a linear quadratic regulator (LQR), which requires recasting the equations of motion in state form. Adjoining the identity $\dot{\mathrm{q}}_{1}=\dot{\mathrm{q}}_{1}$, the state equations can be expressed as

$$
\dot{\mathrm{x}}(t)=A(t) \mathrm{x}(t)+B(t) E_{0} \mathrm{u}_{c}(t)+B(t) D \mathrm{~d}(t)
$$

where $\mathrm{x}=\left[\mathrm{q}_{1}^{T} \dot{\mathrm{q}}_{1}^{T}\right]^{T}$ is the state vector, $\mathrm{u}_{c}=\mathbf{F}_{1 c}$ is the control vector, $\mathbf{d}=\mathbf{Q}_{d}$ is the disturbance vector and

$$
\begin{gathered}
A=\left[\begin{array}{cc}
0 \\
-M_{0}^{-1}\left(M_{a}+M_{v v}-\frac{1}{2} M_{v v}^{\prime}+K-F_{0}^{*}\right) & -M_{0}^{-1}\left(M_{v}+M^{\prime}-M_{v}^{T}\right)
\end{array}\right] \\
B=\left[\begin{array}{c}
0 \\
M_{0}^{-1}
\end{array}\right], D=\left(I-E_{0} E_{0}^{\dagger}\right)
\end{gathered}
$$

are coefficient matrices. It should be noted here that, if the matrix $E_{0}$ is not square, the matrix $D$ is not zero, so that the open-loop control does not annihilate the persistent disturbances completely. As the number of actuator approaches the number of degrees of freedom of the system, the matrix $E_{0}$ tends to become square. When the number of actuators coincides with the number of degrees of freedom the matrix $E_{0}$ is square, in which case the pseudo-inverse becomes an exact inverse and the matrix $D$ reduces to zero.

The state equations, Eq. (50), possess time-varying coefficients and are subject to residual persistent disturbances. Due to difficulties in treating such systems in continuous time, we propose to discretize the state equations in time. Following the usual steps (Ref. 12), the state equations in discrete time can be shown to be

$$
\mathbf{x}_{k+1}=\Phi_{k} \mathbf{x}_{k}+\Gamma_{k} E_{0 k} \mathbf{u}_{c k}+\Gamma_{k} D_{k} \mathbf{d}_{k}, k=0,1, \ldots
$$

where

$$
\begin{aligned}
\mathbf{x}_{k} & =\mathbf{x}(k T), \mathbf{u}_{c k}=\mathrm{u}_{c}(k T), \mathrm{d}_{k}=\mathrm{d}(k T), k=0,1, \ldots \\
\Phi_{k} & =\exp A_{u} T, \Gamma_{k}=\left(\exp A_{k} T-I\right) A_{k}^{-1} B_{k}, k=0,1, \ldots \\
E_{0 k} & =E_{0}(k T), D_{k}=D(k T), k=0,1, \ldots
\end{aligned}
$$


in which $T$ is the sampling period. In view of the above discussion, we assume that the effect of the persistent disturbances has been reduced drastically by the feedforward control, and design the feedback control in its absence. This design is according to a discrete-time LQR with prescribed degree of stability. To this end, we consider the performance measure

$$
J=\mathbf{x}_{N}^{T} P_{N} \mathbf{x}_{N}+\sum_{k=0}^{N-1} e^{2 \alpha k}\left(\mathrm{x}_{k}^{T} Q_{k} \mathbf{x}_{k}+\mathbf{u}_{c k}^{T} R_{k} \mathbf{u}_{c k}\right)
$$

where $P_{N}$ and $Q_{k}$ are symmetric positive semidefinite matrices, $R_{k}$ is a symmetric positive definite matrix, $\alpha$ is a nonnegative constant defining the degree of stability and $N T$ is the final sampling time.

The optimization process using the performance measure given by Eq. (54) can be reduced to a standard discrete-time LQR form by means of the transformation

$$
\hat{\mathrm{x}}_{k}=e^{\alpha k} \mathrm{x}_{k}, \hat{\mathrm{u}}_{c k}=e^{\alpha k} \mathrm{u}_{c k}, \hat{P}_{N}=e^{-2 \alpha N} P_{N}
$$

Multiplying Eqs. (52) through by $e^{\alpha(k+1)}$ using Eqs. $(55 \mathrm{a}, \mathrm{b})$ and ignoring the small perturbing term, we obtain the new state equations

$$
\hat{\mathbf{x}}_{k+1}=e^{\alpha}\left(\Phi_{k} \hat{\mathbf{x}}_{k}+\Gamma_{k} E_{0 k} \hat{\mathbf{u}}_{c k}\right), k=0,1, \ldots, N-1
$$

Similarly, inserting Eqs. (55) into Eq. (54), we obtain the new performance measure

$$
J=\hat{\mathbf{x}}_{N}^{T} \hat{P}_{N} \hat{\mathbf{x}}_{N}+\sum_{k=0}^{N-1}\left(\hat{\mathbf{x}}_{k}^{T} Q_{k} \hat{\mathbf{x}}_{k}+\hat{\mathbf{u}}_{c k}^{T} R_{k} \hat{\mathbf{u}}_{c k}\right)
$$

It can be shown (Ref. 12) that the optimal control law has the form

$$
\hat{\mathbf{u}}_{c k}=G_{k} \hat{\mathbf{x}}_{k}, k=0,1, \ldots, N-1
$$

where $G_{k}$ are gain matrices obtained from the discrete-time Riccati equations

$$
\begin{gathered}
G_{N-i}=-\left(e^{2 \alpha} E_{0, N-i}^{T} \Gamma_{N-i}^{T} \hat{P}_{N+1-i} \Gamma_{N-i} E_{0, N-i}+R_{N-i}\right)^{-1} e^{2 \alpha} E_{0, N-i}^{T} \Gamma_{N-i}^{T} \hat{P}_{N+1-i} \Phi_{N-i} \\
i=1,2, \ldots, N ; \hat{P}=e^{-2 \alpha N} P_{N} \\
\hat{P}_{N-i}=e^{2 \alpha}\left(\Phi_{N-i}+\Gamma_{N-i} E_{0, N-i} G_{N-i}\right)^{T} \hat{P}_{N+1-i}\left(\Phi_{N-i}+\Gamma_{N-1} E_{0, N-i} G_{N-i}\right) \\
+G_{N-i}^{T} R_{N-i} G_{N-i}+Q_{N-i}, i=1,2, \ldots, N ; \hat{P}_{N}=e^{-2 \alpha N} P_{N}
\end{gathered}
$$

Equations (59a) and (59b) are evaluated alternately for $G_{N-1}, \hat{P}_{N-1}, G_{N-2}, \hat{P}_{N-2}, \ldots, G_{0}$, given the final value of $\hat{P}_{N}$.

Inserting the control law, Eqs. (58), into Eqs. (56), we obtain the closed-loop transformed state equations

$$
\hat{\mathrm{x}}_{k+1}=e^{\alpha}\left(\Phi_{k}+\Gamma_{k} E_{0 k} G_{k}\right) \hat{\mathbf{x}}_{k}, k=0,1, \ldots
$$


Then, recalling Eq. (55a) and restoring the persistent disturbance term, the closed-loop state equations for the original system can be written in the form

$$
\mathrm{x}_{k+1}=\left(\Phi_{k}+\Gamma_{k} E_{0 k} G_{k}\right) \mathrm{x}_{k}+\Gamma_{k} D_{k} \mathrm{~d}_{k}, k=0,1, \ldots
$$

\section{NUMERICAL EXAMPLE}

The example involves the flexible space robot shown in Fig. 1. Numerical values for the system parameters are as follows:

$$
\begin{aligned}
L_{1} & =1 \mathrm{~m}, d_{1}=0.5 \mathrm{~m}, L_{2}=L_{3}=5 m, L_{4}=1.66 \mathrm{~m} \\
m_{1} & =10 \mathrm{~kg}, m_{2}=m_{3}=1 \mathrm{~kg}, m_{4}=0.1 \mathrm{~kg} \\
J_{1} & =20 \mathrm{kgm}^{2}, J_{2}=3 \mathrm{kgm}^{2}, E I_{2}=E I_{3}=122.28 \mathrm{Nm}^{2}
\end{aligned}
$$

The quasi-comparison functions for the flexible arm were chosen as a linear combination of clamped-free and clamped-clamped shape functions. Both families of shape functions have the functional form

$$
\phi_{i}=\frac{1}{\sqrt{L}}\left[\cosh \lambda_{i} x / L-\cos \lambda_{i} x / L-\sigma_{i}\left(\sinh \lambda_{i} x / L-\sin \lambda_{i} x / L\right)\right], i=1,2, \ldots, n
$$

The values of $\lambda_{i}$ and $\sigma_{i}$ are given in Table 1. They correspond to two clamped-free and three clamped-clamped shape functions, for a total of $n=5$ for each flexible arm.

The initial and final end-effector positions are defined by

$$
\begin{aligned}
& X_{i}=9.75 \tau \mathrm{m}, Y_{i}=1.914 \mathrm{~m}, \theta_{4 f}=0 \mathrm{rad} \\
& X_{f}=5.000 \mathrm{~m}, Y_{f}=1.914 \mathrm{~m}, \theta_{4 f}=\pi / 2 \mathrm{rad}
\end{aligned}
$$

and we note that the path from the initial to the final position represents a straight-line translation, while the orientation undergoes a $90^{\circ}$ change. In terms of time, the translational and rotational accelerations represent one-cycle sinusoidal curves.

The maneuver time is $t_{f}=2.5 \mathrm{~s}$. The zero-order actuator forces and torques to carry out the maneuver are shown in Fig. 3.

The control of the elastic motions and the perturbations in the rigid-body motions was extended to $t=4 \mathrm{~s}$. Not that for $2.5 \mathrm{~s}<t<4 \mathrm{~s}$ the system is time-invariant, during which time the control gains can be regarded as constant. The weighting matrices in the performance measure are

$$
Q_{k}=10 I, R_{k}=I, P_{N}=10 I
$$

The degree of stability constant is $\alpha=0.1$. Moreover, the samping period is $T=0.01 \mathrm{~s}$ and the number of time increments is $N=350$.

Time-lapse plots of the uncontrolled and controlled robot configuration are shown in Figs. 4a and $4 \mathrm{~b}$, respectively, at the instants $0,1,1.5$ and $2.5 \mathrm{~s}$. Figures 5 and 6 show time histories of the 
errors in the end-effector position. The discrete-time open-loop and closed-loop poles are given in Tables 2 and 3 . For comparison, Fig. 7 shows the time history of the errors and Table 4 gives the closed-loop poles for $\alpha=1$.

It should be pointed out that the actuator dynamics is also included in the formulation and the numerical results, but the effect turned out to be small.

\section{CONCLUSIONS}

An orderly kinematic synthesis in conjunction with the Lagrangian approach permits the derivation of the equations of motion for an articulated multibody system, such as those describing the dynamical behavior of a flexible space robot, without the imposition of constraints. The equations are nonlinear and of relatively high order. A perturbation approach permits the separation of the problem into a zero-order problem (in a perturbation sense) for the rigid-body maneuvering of the space robot and a first-order problem for the control of the elastic motions and the perturbations from the rigid-body motions. The robot mission consists of carrying a payload over a prescribed trajectory and placing it in a cerrtain orientation relative to the inertial space. This represents the zero-order problem and the control can be carried out open loop. The firstorder equations defining the first-order problem (in a perturbation sense) are linear, time-varying, of high-order and subject to persistent disturbances. The persistent disturbances are treated by means of feedforward control. All other disturbances are controlled closed loop, with the feedback control being designed by means of discrete-time LQR theory with prescribed degree of stability. In a numerical example, the controls derived by the perturbation approach are found to work satisfactorily when applied to the original nonlinear system.

\section{REFERENCES}

1. Book, W.J., Maizza-Neto, A., and Whitney, D.E.: Feedback Control of Two Beam, Two Joint Systems with Distributed Flexibility. ASME Journal of Dynamic Systems, Measurement, and Control, vol. 97, Dec. 1975, pp.424-431.

2. Kozel, D. and Koivo, A.J.: A General Force/Torque Relationship and Kinematic Representation for Flexible Link Manipulators. Journal of Robotic Systems, vol. 8, no. 4, 1991, pp. $531-556$.

3. Wang, D. and Vidyasagar, M.: Control of a Class of Manipulators with a Single Flexible Link-Part I: Feedback Linearization. ASME Journal of Dynamic Systems, Measurement, and Control, vol. 113, Dec. 1991, pp. 655-661.

4. Nathan, P.J., and Singh, S.N.: Sliding Mode Control and Elastic Mode Stabilization of a Robotic Arm with Flexible Links. ASME Journal of Dynamic Systems, Measurement, and Control, vol. 113, Dec. 1991. 
5. Umetani, Y. and Yoshida, K.: Continuous Path Control of Space Manipulators Mounted on OMV. Acta Astronautica, vol. 15, no. 12, 1987, pp. 981-986.

6. Nenchev, D., Umetani, Y., and Yoshida, K.: Analysis of a Redundant Free-Flying Spacecraft/Manipulator System., IEEE Transactions on Robotics and Automation, vol. 8, no. 1, 1992, pp. 1-6.

7. Nakamura, Y. and Mukherjee, R.: Nonholonomic Path Planning of Space Robots via a Bidirectional Approach. IEEE Transactions on Robotics, and Automation, vol. 7, no. 4, 1991, pp. 500-514.

8. Usoro, P.B., Nadira, R., Mahil, S.S.: Control of Lightweight Flexible Manipulators: A Feasibility Study. Proceedings of the American Control Conference, vol. 3, San Diego, CA, June 1984, pp. 1209-1216.

9. Meirovitch, L., Stemple, T., and Kwak, M.K.: Dynamics and Control of Flexible Space Robots. Proceedings of the CSME Mechanical Engineering Forum, Toronto, Ontario, 1990.

10. Meirovitch, L.: Hybrid State Equations of Motion for Flexible Bodies in Terms of QuasiCoordinates. Journal of Guidance, Control, and Dynamics, vol. 14, no. 5, 1991, pp. 10081013.

11. Meirovitch, L. and Kwak, M.K.: Convergence of the Classical Rayleigh-Ritz Method and the Finite Element Method. AIAA Journal, vol. 28, no. 8, 1990, pp. 1509-1516.

12. Kuo, B.C.: Digital Control Systems, SRL Publishing Co., Champaign, IL, 1977.

Table 1. Shape Function Coefficients

\begin{tabular}{|c|c|c|}
\hline$i$ & $\lambda_{i}$ & $\sigma_{i}$ \\
\hline 1 & 1.87510407 & 0.734095514 \\
\hline 2 & 4.69409113 & 1.018467319 \\
\hline 3 & 7.85475744 & 0.999224497 \\
\hline 4 & 10.99554073 & 1.000033553 \\
\hline 5 & 14.13716839 & 0.999998550 \\
\hline
\end{tabular}

Table 2. Discrete-Time Open-Loop Poles

\begin{tabular}{|c|c|c|c|l|l|}
\hline No. & Pole Location & Mag. & No. & Pole Location & Mag. \\
\hline 1,2 & $-0.840 \pm 0.543 i$ & 1.000 & 17,18 & $0.991 \pm 0.135 i$ & 1.000 \\
\hline 3,4 & $-0.778 \pm 0.629 i$ & 1.000 & 19,20 & $0.994 \pm 0.107 i$ & 1.000 \\
\hline 5,6 & $-0.700 \pm 0.714 i$ & 1.000 & 21,22 & 1.000 & 1.000 \\
\hline 7,8 & $-0.690 \pm 0.724 i$ & 1.000 & 23,24 & 1.000 & 1.000 \\
\hline 9,10 & $0.586 \pm 0.8101$ & 1.000 & 25,26 & 1.000 & 1.000 \\
\hline 11,12 & $0.629 \pm 0.7781$ & 1.000 & 27,28 & 1.000 & 1.000 \\
\hline 13,14 & $0.902 \pm 0.4311$ & 1.000 & 29,30 & 1.000 & 1.000 \\
\hline 15,16 & $0.921 \pm 0.390 i$ & 1.000 & 31,32 & 1.000 & 1.000 \\
\hline
\end{tabular}


Table 3. Discrete-Time Closed-Loop Poles

\begin{tabular}{|c|l|c|c|l|l|}
\hline No. & Pole Location & Mag. & No. & Pcle Location & Mag. \\
\hline 1.2 & $-0.169 \pm 0.546 i$ & 0.572 & 18.19 & $0.803 \pm 0.976 \times 10^{-1} i$ & 0.809 \\
\hline 3 & $0.493 \times 10^{-2}$ & 0.005 & 20 & 0.805 & 0.805 \\
\hline 4 & $0.120 \times 10^{-1}$ & 0.012 & 21 & 0.807 & 0.807 \\
\hline 5 & 0.125 & 0.125 & 22.23 & $0.814 \pm 0.362 \times 10^{2} \mathrm{i}$ & 0.814 \\
\hline 6 & 0.204 & 0.204 & 24.25 & 0.817 & 0.817 \\
\hline 7.8 & $0.302 \pm 0.1481$ & 0.336 & 26 & 0.817 & 0.817 \\
\hline 9.10 & $0.454 \pm 0.4931$ & 0.670 & 27 & 0.819 & 0.819 \\
\hline 11.12 & $0.468 \pm 0.323 i$ & 0.569 & 28.29 & $0.821 \pm 0.366 \times 10^{-2}$ & 0.821 \\
\hline 13,14 & $0.536 \pm 0.500 i$ & 0.733 & 30 & 0.822 & 0.822 \\
\hline 15.16 & $0.749 \pm 0.860 \times 10 \mathrm{i}$ & 0.754 & 31 & 0.822 & 0.822 \\
\hline 17 & 0.792 & 0.792 & 32 & 0.827 & 0.827 \\
\hline
\end{tabular}

Table 4. Discrete-'Time Closed-Loop Poles for $\alpha=1$

\begin{tabular}{|l|l|l|l|l|l|}
\hline No. & Pole Location & Mag. & No. & Pole Location & Mag. \\
\hline 1 & -0.566 & 0.566 & 17,18 & $0.139 \pm 0.844 \times 1 \sigma^{2} i$ & 0.139 \\
\hline 2.3 & $-0.160 \pm 0.186 i$ & 0.246 & 19,20 & $0.150 \pm 0.022 i$ & 0.152 \\
\hline 4,5 & $-0.109 \pm 0.275 i$ & 0.296 & 21,22 & $0.187 \pm 0.145 i$ & 0.236 \\
\hline 6.7 & $0.062 \pm 0.088 i$ & 0.108 & 23.24 & $0.198 \pm 0.288 \times 10^{i} i$ & 0.200 \\
\hline 8 & $-0.177 \times 10^{-1}$ & 0.018 & 25 & 0.251 & 0.251 \\
\hline 9.10 & $0.779 \times 10^{2} \pm 0.209 i$ & 0.209 & 26,27 & $0.252 \pm 0.180 i$ & 0.310 \\
\hline 11,12 & $0.072 \pm 0.088 i$ & 0.114 & 28.29 & $0.279 \pm 0.490 i$ & 0.564 \\
\hline 13,14 & $0.118 \pm 0.016 i$ & 0.119 & 30.31 & $0.328 \pm 0.148 i$ & 0.360 \\
\hline 15,16 & $0.132 \pm 0.920 \times 10^{2} i$ & 0.132 & 32 & 0.430 & 0.430 \\
\hline
\end{tabular}

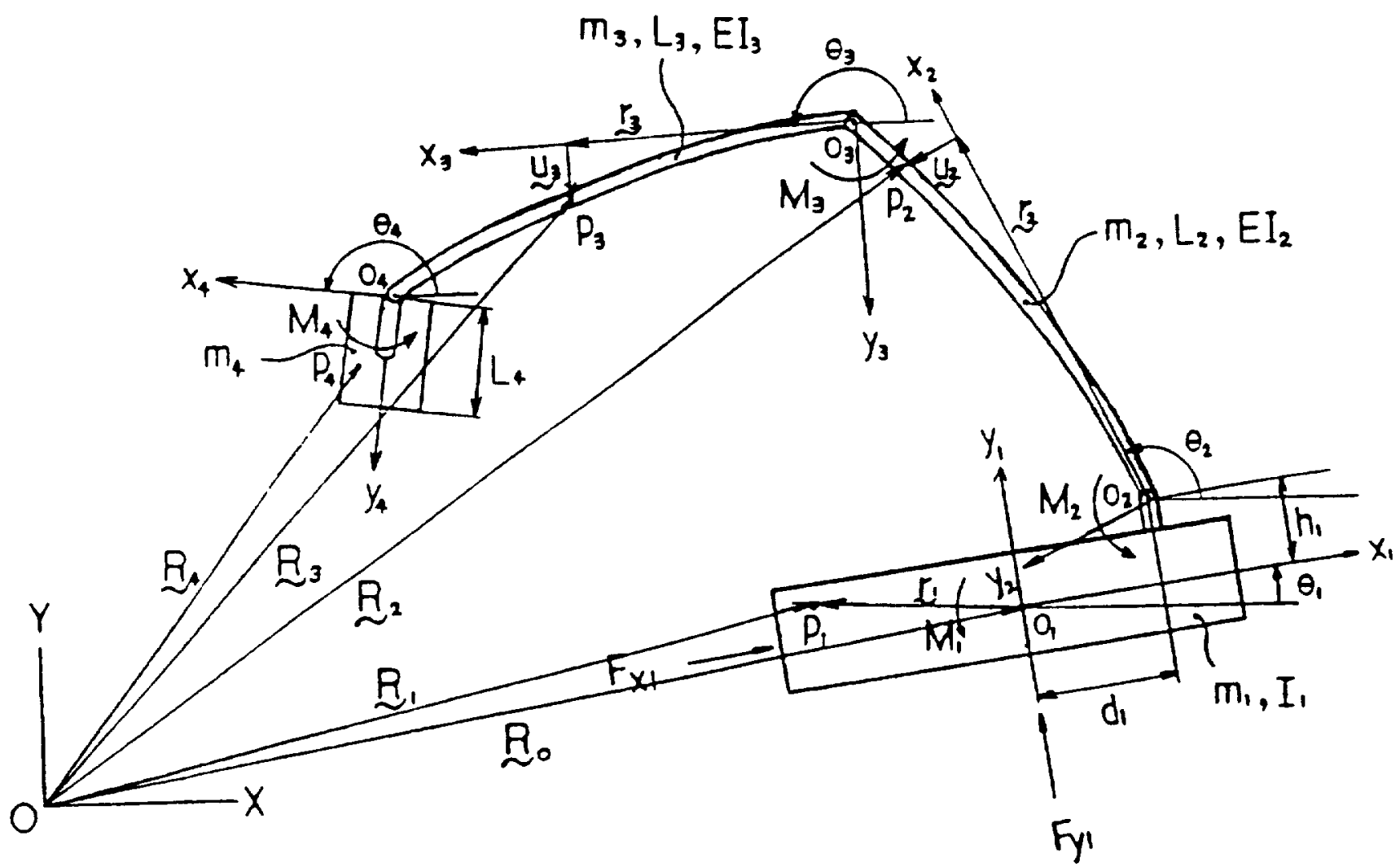

Figure 1. Flexible Space Robot 


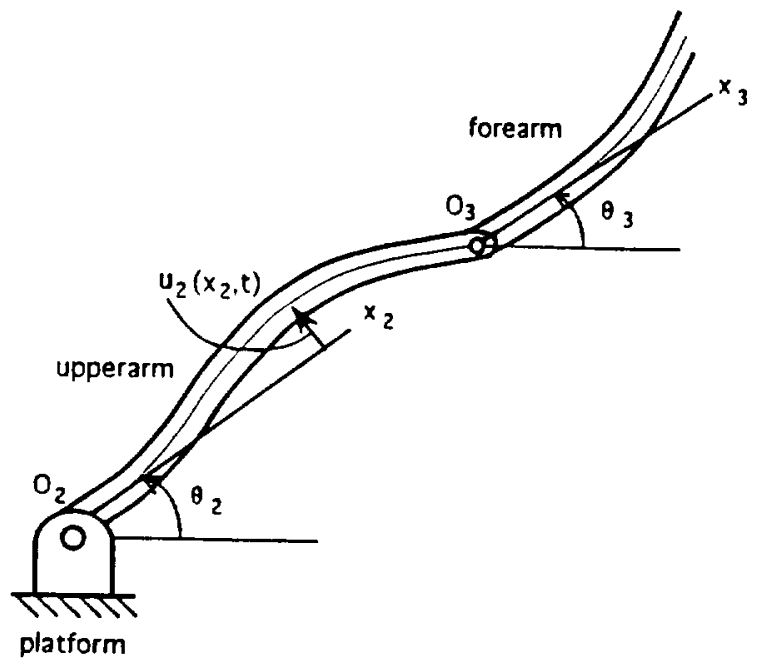

Figure 2. Definition of Body Axes

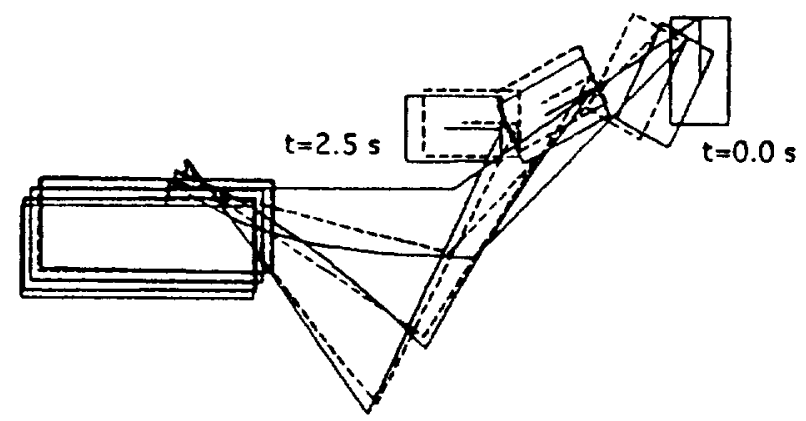

Figure 4a. Uncontrolled Maneuver

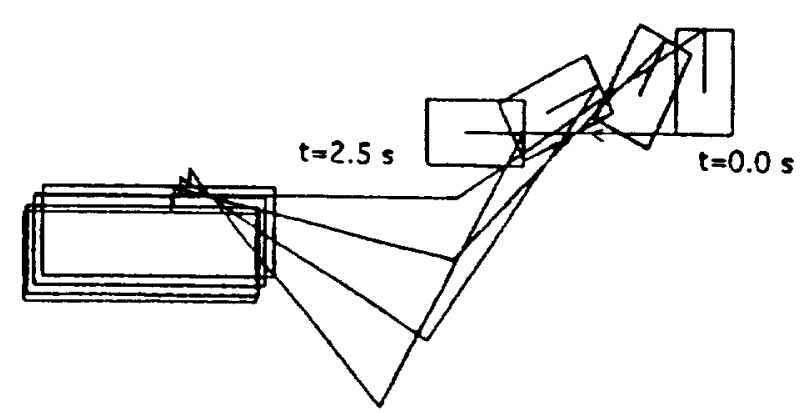

Figure 4b. LQR-Controlled Maneuver

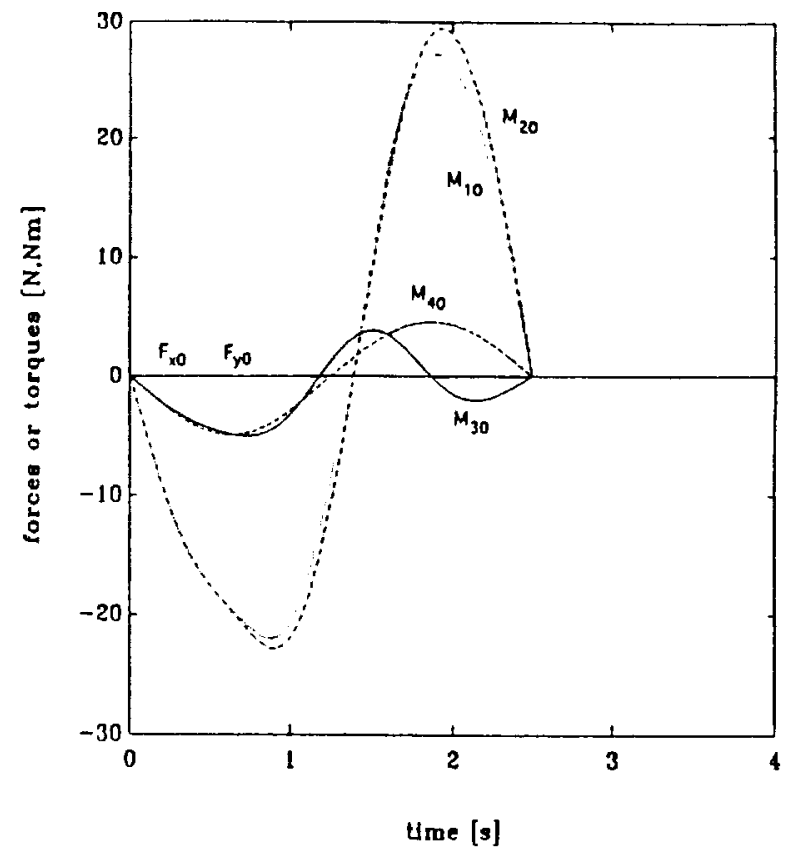

Figure 3. Zero-Order Forces and Torques

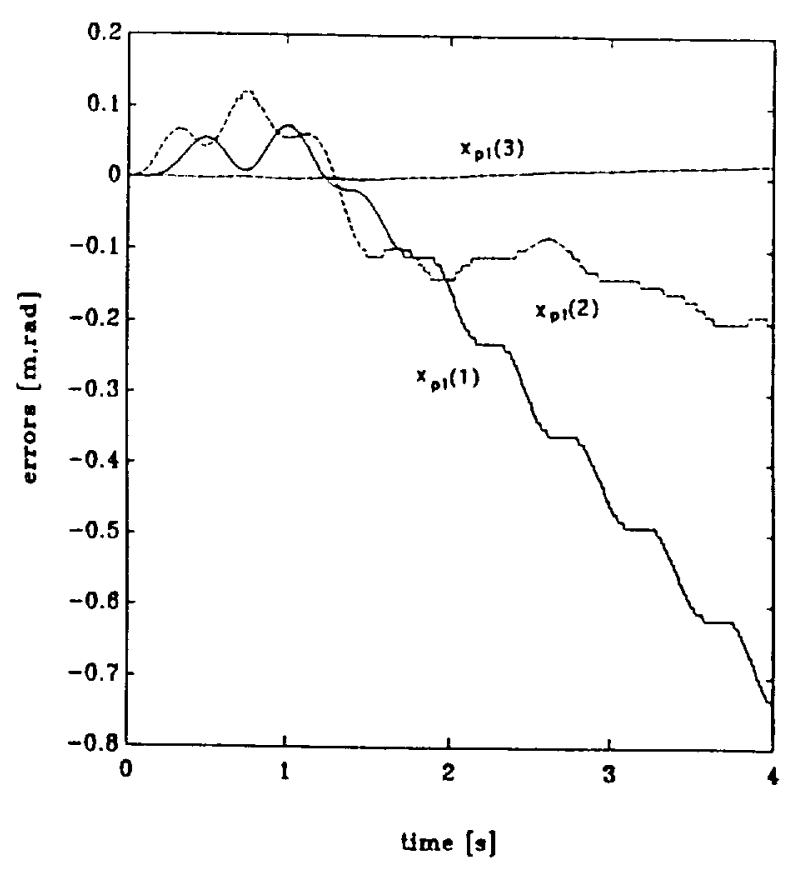

Figure 5. Uncontrolled End-Effector Position Errors 


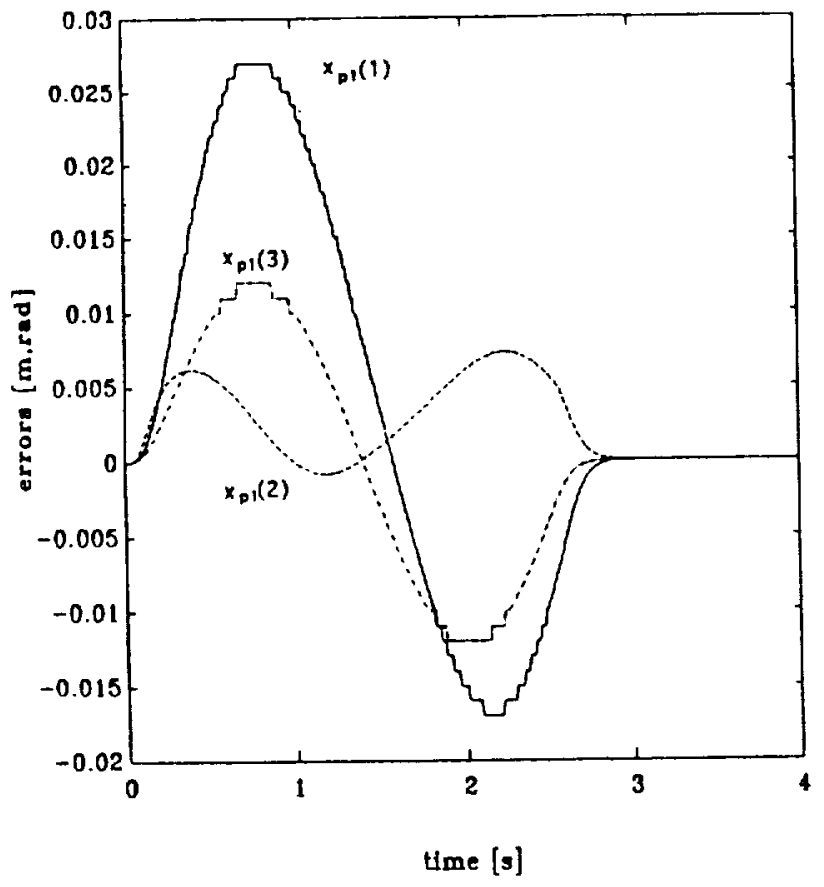

Figure 6. LQR-Controlled End-Effector Position Errors for $\alpha=0.1$

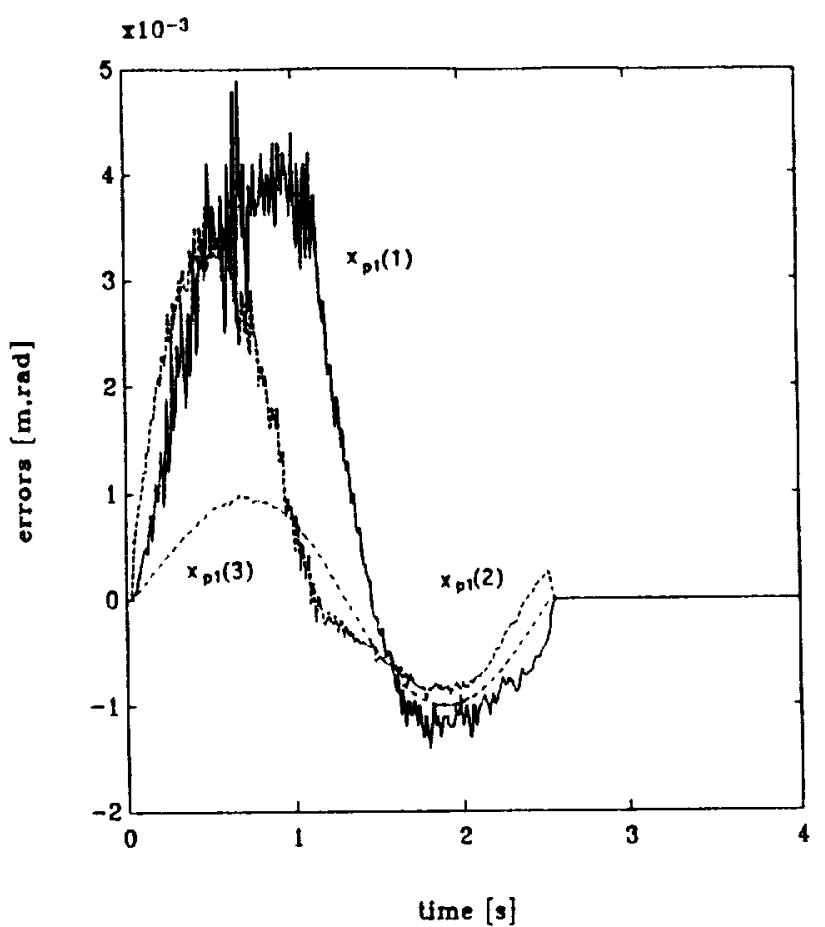

Figure 7. LQR-Controlled End-Effector Position Errors for $\alpha=1$ 